\title{
Filmes finos de óxido de zinco depositados por RF-magnetron sputtering na presença de oxigênio
}

\author{
Fine films of zinc oxide deposited by RF-magnetron sputtering in the \\ presence of oxygen
}

William Emanuel Silva Santos Viana ${ }^{1}$, Marcelo Banto Pisani ${ }^{1}$, Gesil Sampaio Amarante Segundo ${ }^{1}$, Nestor Santos Correia ${ }^{1}$

\section{RESUMO}

Neste trabalho são estudados e apresentados os resultados da deposição e caracterização de filmes finos de óxido de zinco ( $\mathrm{ZnO})$, obtidos através da técnica de pulverização catódica por plasma de radiofrequência (do inglês $\mathrm{RF}$ sputtering) realizadas à temperatura ambiente (entre $25^{\circ} \mathrm{C}$ a $30^{\circ} \mathrm{C}$ ) sobre substratos de vidro. O objetivo deste trabalho é o de desenvolver uma sequência de deposição controlada dos filmes, variando-se e correlacionando-se todos os parâmetros de processo envolvidos na mesma, tais como: pressão de processo, taxa e tempo de deposição, vazão e composição dos gases de processo, tensão de auto-polarização entre alvo e substrato, a fim de se garantir a reprodutibilidade do processo, obtendo por meio deste, filmes com espessura e fase cristalina bem definidas, de alta transparência na faixa do visível visando principalmente a aplicação em dispositivos optoeletrônicos. Os filmes foram caracterizados através das técnicas de difração de raios-X (DRX) para obtenção das fases cristalinas, da espectrofotometria na faixa do UV-visível para determinar os níveis de transmitância e o band gap óptico dos filmes, da microscopia eletrônica de varredura (MEV) para revelar a morfologia dos filmes e da perfilometria mecânica para medir as respectivas espessuras. Os resultados obtidos evidenciaram bons resultados para transmitância na faixa do UV-visível (superior a $90 \%$ ). O band gap óptico de todas está de acordo com os valores esperados para filmes de $\mathrm{ZnO}$. A difração de raios-X juntamente com as imagens de MEV revelaram a forte influência da presença de oxigênio na mistura gasosa de deposição sobre a qualidade da morfologia e cristalinidade das amostras.

Palavras-chave: Óxido de zinco; Filmes finos; Magnetron Sputtering; Cristalinidade.

\begin{abstract}
In this work the results of the deposition and characterization of thin films of zinc oxide ( $\mathrm{ZnO}$ ), obtained by means of sputtering by radiofrequency plasma (RF sputtering), are carried out at room temperature $\left(25^{\circ} \mathrm{C}\right.$ to $\left.30^{\circ} \mathrm{C}\right)$ on glass substrates. The objective of this work is to develop a controlled deposition sequence of the films, varying and correlating all the process parameters involved, such as: process pressure, rate and deposition time, flow rate and gas composition of process, self bias between target and substrate, in order to guarantee the reproducibility of the process, obtaining through this, films with thickness and crystalline phase well defined, of high transparency in the band of the visible aiming mainly the application in devices optoelectronics. The films were characterized by X-ray diffraction (XRD) techniques to obtain the crystalline phases, spectrophotometry in the UV-visible band to determine transmittance levels and optical band gap of films, scanning electron microscopy (SEM) to reveal the morphology of the films and the mechanical profilometry to measure the respective thicknesses. The results obtained showed good results for transmittance in the UV-visible range (greater than 90\%). The optical band gap of all conforms to the expected values for $\mathrm{ZnO}$ films. The X-ray diffraction together with SEM images revealed the strong influence of the presence of oxygen in the gas deposition mixture on the quality of the morphology and crystallinity of the samples.
\end{abstract}

Keywords: Zinc oxide; Thin films; Magnetron Sputtering; Crystallinity.

'Universidade Estadual de Santa Cruz - Ilhéus (BA) - Brasil

Autor correspondente: William Emanuel Silva Santos Viana - Universidade Estadual de Santa Cruz - Rodovia Jorge Amado, Km 16, Bairro Salobrinho. CEP: 45662-900 - Ilhéus (BA) - Brasil

Email: will.uesc.fis@gmail.com

Recebido: 18/07/2017 Aceito: 22/11/2017 


\section{INTRODUÇÃO}

$\mathrm{O}$ óxido de zinco $(\mathrm{ZnO})$ é um material semicondutor, com uma faixa de energia inacessível, chamada de banda proibida - band gap - com valores, à temperatura de $300 \mathrm{~K}$, entre $3,0 \mathrm{eV}$ e 3,4 eV $\mathrm{eV}^{(1)}$. $\mathrm{Na}$ forma monocristalina, à temperatura ambiente apresenta estrutura hexagonal wurtzita, com orientação preferencial (002), o band gap pode ser controlado alterando-se a estequiometria entre o zinco e o oxigênio, ou através de adição de outros elementos químicos à estrutura cristalina do material (dopagem).

Em meados de 1950 ocorreu uma redescoberta das potenciais aplicações do $\mathrm{ZnO}$. Percebeu-se que este tinha muitas novas propriedades interessantes como a semicondutividade, a piezeletricidade, a luminescência, a absorção de luz no ultravioleta (UV), entre outras.

Durante os anos 1960, observou-se o elevado coeficiente piezoelétrico deste material, o que levou ao desenvolvimento de transdutores piezoelétricos baseados no mesmo, como os medidores de tensão sensível e sensores de pressão, uma tecnologia que é utilizada até os dias de hoje. Extensivas investigações foram realizadas sobre as propriedades semicondutoras do $\mathrm{ZnO}$, incluindo o estudo da estrutura de bandas de energia, propriedades excitônicas, efeitos de elétrons e buracos, propriedades elétricas e de geração e propagação fônons ${ }^{(2)}$.

A utilização do $\mathrm{ZnO}$ como filme fino ganhou muito destaque a partir dos últimos desenvolvimentos tecnológicos, e tem grande importância entre os óxidos condutores transparentes (TCOs, do inglês Transparent Conductive Oxides) devido a sua alta transmitância na faixa do visível (superior a 80\%) e baixa resistividade ${ }^{(3)}$.

As técnicas de fabricação filmes finos de $\mathrm{ZnO}$ mais utilizadas são: spray-pirólise, laser pulsado (PLD), CVD (chemical vapor deposition) e magnetron sputtering (MS) de corrente contínua pulsada (DC pulsada) e rádio frequência $(\mathrm{RF})$. Dentre estas o sputtering se destaca, pois apresenta boa reprodutibilidade e a possibilidade de deposições uniformes em grandes áreas ${ }^{(4)}$.

Durante a deposição dos filmes finos de $\mathrm{ZnO}$, as propriedades finais estão fortemente ligadas aos parâmetros de processamento utilizados. Quando utilizada a técnica magnetron sputtering, como por exemplo, a potência elétrica fornecida pela fonte de RF, a vazão de argônio (Ar) e de oxigênio $\left(\mathrm{O}_{2}\right)$, a temperatura dentro da câmara e no substrato, a pressão de processo, e o tempo de deposição são os principais parâmetros de processo $^{(5)}$.

Este trabalho consistiu em estudar a influência da mistura entre os gases de processo $\left(\mathrm{Ar} \mathrm{e} \mathrm{O}_{2}\right)$ sobre as propriedades estruturais, óticas dos filmes finos de óxido de zinco depositados sobre vidro por Magneton Sputtering(6).

Tabela 1: Parâmetros de deposição.

\section{MATERIAIS E MÉTODOS}

Antecedendo o experimento, foram realizadas limpezas das superfícies dos materiais a serem utilizados, para salvaguardar a qualidade das mesmas. Como substratos, foram escolhidas lâminas de vidro retangulares de microscopia.

Inicialmente as lâminas foram limpas em banho de ultrassom com detergente por $30 \mathrm{~min}$, e após limpo com álcool isopropílico, colocadas no interior da câmara de deposição como mostra a Fig. 1. Após montar o aparato experimental, foi realizada a limpeza das superfícies das amostras durante $10 \mathrm{~min}$, com plasma de argônio para a retirada de possíveis impurezas das superfícies, a uma pressão de 5,0 × 10-2 mbar e self bias de $1200 \mathrm{~V}$. Os parâmetros para o procedimento de deposição dos filmes estão listados na Tabela 1.

Os parâmetros que constam na Tabela 1 foram escolhidos a partir de investigações prévias no Laboratório de Filmes Finos (LFF) da UESC. Antes de contemplar a fase da deposição dos filmes variando a proporção de $\mathrm{O}_{2}$ e Ar (Tabela 2), foram testados alguns outros parâmetros como: distância entre o alvo e o substrato e pressão de trabalho, com o objetivo de obter uma janela de operação que favorecesse maiores taxas de deposição, mantendo uma deposição uniforme ao longo de todo o substrato.

Mantendo a pressão de processo constante, foi alterada a concentração parcial de oxigênio na mistura gasosa utilizada durante a deposição dos filmes. Os difratogramas na Fig. 2 apresentam a influência desse parâmetro de processo sobre a qualidade da cristalinidade dos filmes depositados.

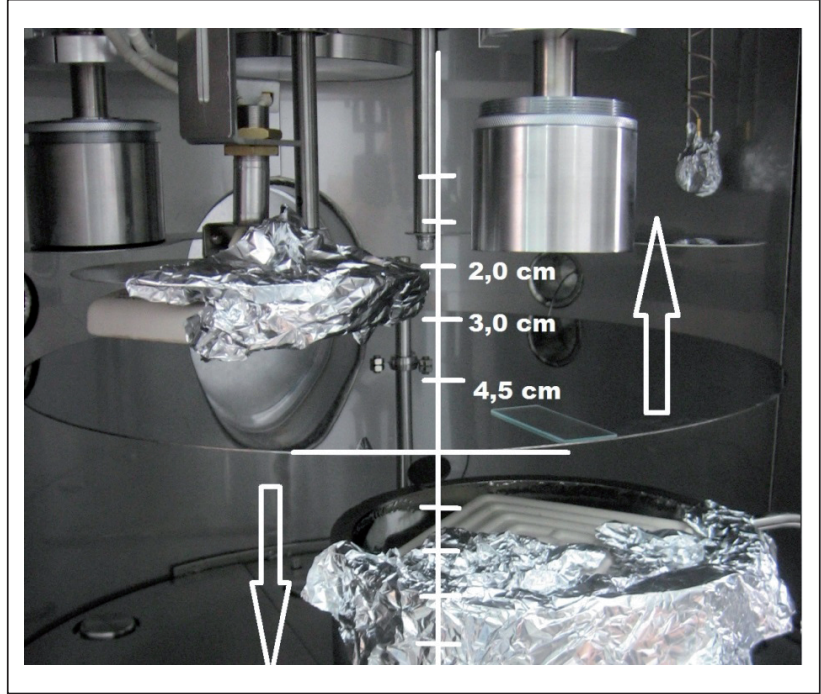

Figura 1: Interior de câmara de deposição com destaque para o posicionamento entre o alvo e substrato, localizados a uma distância ajustável.

\begin{tabular}{|c|c|c|c|c|c|}
\hline Potência da fonte RF & $\begin{array}{c}\text { Distância } \\
\text { alvo-substrato }\end{array}$ & Self Bias & $\begin{array}{c}\text { Pressão de } \\
\text { processo }\end{array}$ & $\begin{array}{c}\text { Temperatura } \\
\text { do substrato }\end{array}$ \\
\hline $100 \mathrm{~W}$ & $45 \mathrm{~mm}$ & 330 Volts & $7,3 \times 10^{-3} \mathrm{mbar}$ & $10 \mathrm{~min}$ \\
\hline
\end{tabular}


Tabela 2: Valores dos parâmetros de processo utilizados para estudo da influência da concentração de oxigênio no processo de deposição.

\begin{tabular}{|c|c|c|c|c|}
\hline Amostra & Potência $(\mathbf{W})$ & Vazão de Ar (sccm) & Vazão de $\mathbf{O}_{2}(\mathbf{s c c m})$ & Proporção de $\mathbf{O}_{2}(\%)$ na mistura \\
\hline P3.20 & 100 & 20 & 0 & 0 \\
\hline P3.16 & 100 & 16 & 4 & 20 \\
\hline P3.14 & 100 & 14 & 6 & 30 \\
\hline P3.10 & 100 & 10 & 10 & 50 \\
\hline P3.06 & 100 & 6 & 14 & 70 \\
\hline
\end{tabular}

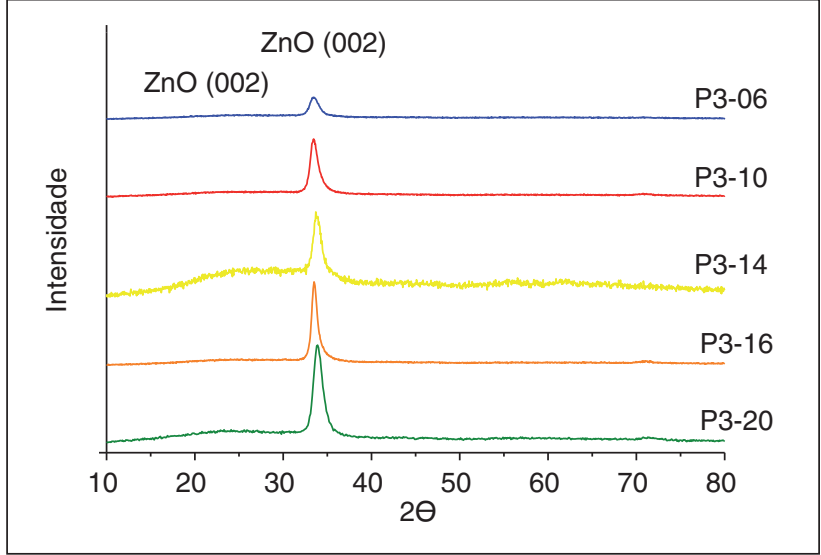

Figura 2: Espectros de Difração de Raios-X, dos filmes de ZnO depositados com diferentes concentrações de oxigênio.

Foram utilizadas as técnicas de espectrofotometria na faixa do UV-visível para a avaliação do band gap, e a microscopia eletrônica de varredura - MEV para realizar imagens e analisar a morfologia dos filmes depositados.

\section{RESULTADOS E DISCUSSÕES}

Mantendo a pressão de processo constante, foi alterada a concentração parcial de oxigênio na mistura gasosa utilizada durante a deposição dos filmes. Os difratogramas da Fig. 2 apresentam a influência desse parâmetro de processo sobre a qualidade da cristalinidade dos filmes depositados.

Observa-se então que quanto menor a proporção de oxigênio na mistura dos gases de processo, maior a intensidade dos picos do plano cristalino (002) para $2 \theta=34^{\circ}$.

A variação da concentração de oxigênio na mistura de gases do processo de deposição também leva a alteração das propriedades óticas que foram estudadas por meio da espectrofotometria na faixa do UV- visível. Através da técnica é possível obter os gráficos de intensidade de fótons absorvida pelo filme ou de porcentagem de fótons que é transmitida (Fig. 3), a partir dos dados que geram esses gráficos é possível obter o valor para o band gap dos filmes de $\mathrm{ZnO}$ depositados.

Para obter o valor da banda proibida foi feita a extrapolação da reta tangente à curva de absorbância por energia, no intervalo de valores no qual ocorre o início da absorção, como mostra a Fig. 4.

Com o aumento da pressão de processo utilizando com o gás argônio (amostra P3-20, Tabela 3), há um aumento da

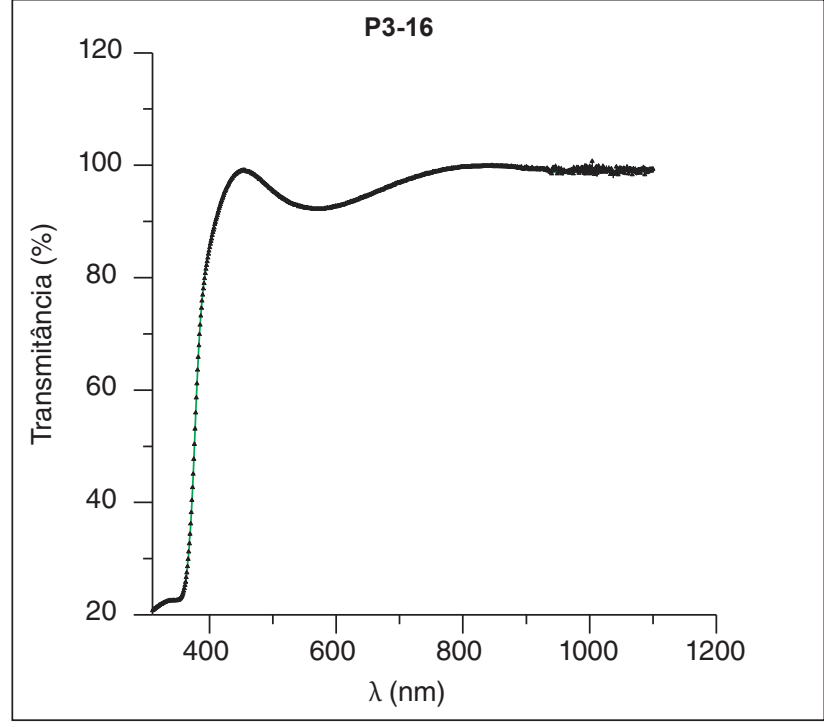

Figura 3: Gráfico de transmitância da amostra P3-16.

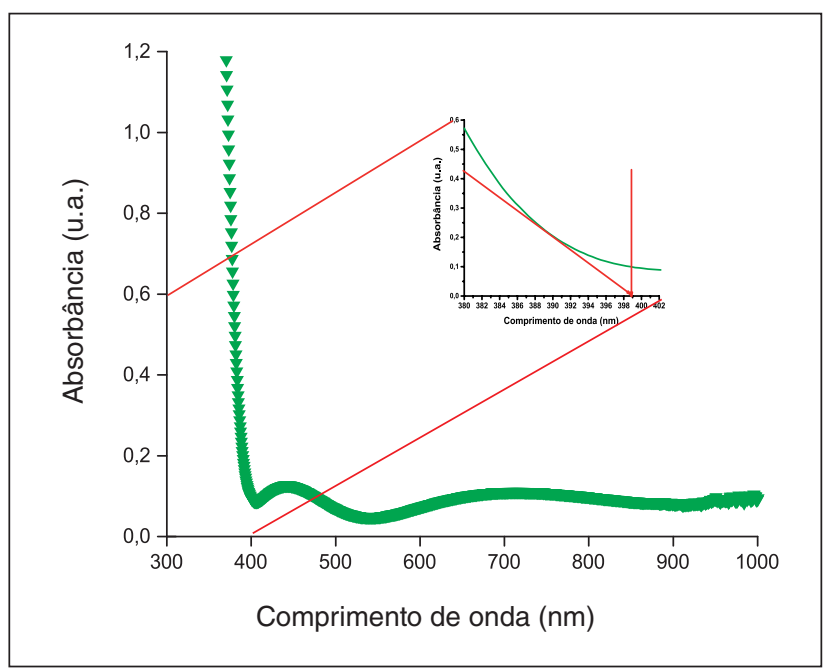

Figura 4: Gráfico de absorbância versus comprimento de onda com destaque para região de interesse onde ocorre o início na absorção.

energia dos íons e consequentemente o aumento da energia dos átomos que se depositam sobre o substrato. Tais efeitos são responsáveis pelo aparecimento de uma direção de crescimento preferencial, conhecida como orientação c, na qual os átomos se empilham perpendicularmente à superfície do substrato, formando estruturas colunares como se pode observar na microscopia eletrônica de varredura, na Fig. 5. 
Tabela 3: Valores da proporção de oxigênio, da transmitância e do band gap.

\begin{tabular}{|c|c|c|c|}
\hline Amostra & Proporção de $\mathrm{O}_{2}(\%)$ na mistura & Transmitância (\%T) em $\lambda=550$ nm & Band gap (eV) \\
\hline P3.20 & 0 & 98,6 & 3,09 \\
\hline P3.16 & 20 & 92,5 & 3,04 \\
\hline P3.14 & 30 & 97,6 & 3,09 \\
\hline P3.10 & 50 & 99,2 & 3,09 \\
\hline P3.06 & 70 & 95,6 & 3,05 \\
\hline
\end{tabular}

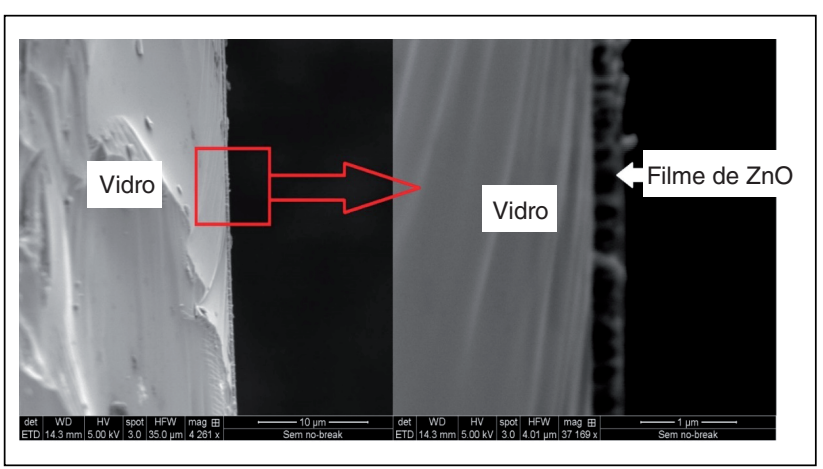

Figura 5: Imagem de MEV do filme P3-20 apresentando estruturas colunares

\section{CONCLUSÃO}

A partir das análises de DRX foi possível ver, através da intensidade dos picos da fase wurtzita, que o oxigênio presente durante a deposição altera a cristalinidade do filme. O parâmetro de processo que sofreu variação foi a concentração de oxigênio na mistura de gases $\left(\mathrm{Ar}+\mathrm{O}_{2}\right)$ utilizada. Os difratogramas revelam que quanto menor a proporção de oxigênio na mistura maior é a intensidade referente aos planos cristalinos (002) com $2 \theta=34^{\circ}$, ou seja, quanto maior a quantidade de oxigênio durante o processo de deposição menor a qualidade cristalina dos filmes finos de $\mathrm{ZnO}$ depositados sobre o vidro, propriedade esta também comprovada pela imagem de MEV, onde é possível visualizar a estrutura colunar.

Todos difratogramas foram realizados utilizando as mesmas condições de medida para todas as amostras, e as mesmas possuem aproximadamente a mesma espessura e tipo de substrato, o que descarta a possibilidade de que as diferentes intensidades do pico (002) da difração de raios-X estarem relacionadas com a quantidade de material analisado ou de estarem ligadas a outro parâmetro de processo não controlado.

A análise dos resultados revela que há uma pequena influência da concentração de oxigênio como gás de processo no valor do band gap e da transmitância dos filmes de óxido de zinco depositados sobre vidro. A inexistência de uma variação direta entre o parâmetro de processo e as características óticas não invalidam os resultados. A utilização desta técnica em conjunto com outras caracterizações sugere que o material depositado é realmente uma fase pura e estequiométrica do $\mathrm{ZnO}$, mostrando valores de band gap dentro dos esperados e apresentados pela literatura para este material $3,0 \mathrm{eV}-3,4 \mathrm{eV}$.

\section{AGRADECIMENTOS}

A CAPES pela bolsa; aos professores, colegas e funcionários da UESC que contribuíram na confecção deste trabalho.

\section{REFERÊNCIAS:}

1. ÖZGÜR, U.; ALIVOV,Y.I.; LIU, C.; TEKE, A.; RESHCHIKOV,M.A.; DOGAN, S.; AVRUTIN, V.; CHO, S.-J.; MORKOÇ, H.; A comprehensive review of $\mathrm{ZnO}$ materials and devices, Journal of Applied Physics, vol. 98, no. 4, p. 41301, 2005.

2. $\mathrm{LI}, \mathrm{Z}$.; GAO, W.; ZnO thin films with $\mathrm{DC}$ and $\mathrm{RF}$ reactive sputtering, Materials Letters, vol. 58, no. 7-8, pp. 1363-1370, 2004.

3. CARCIA, P.F.; MCLEAN, R.S.; REILLY, M.H.; NUNES, G.; Transparent $\mathrm{ZnO}$ thin-film transistor fabricated by if magnetron sputtering, Applied Physics Letters, vol. 82, no. 7, p. 1117, 2003.

4. BIN LEE, J.; KIM, H.J.; KIM, S.G.; HWANG, C.S.; HONG, S.-H.; SHIN, Y.H.; LEE N.H.;

5. Deposition of $\mathrm{ZnO}$ thin films by magnetron sputtering for a film bulk acoustic resonator, Thin Solid Films, vol. 435, no. 1-2, pp. 179-185, 2003.

6. WANG, Z.A.; CHU, J.B.; ZHU, H.B.; SUN, Z.; CHEN, Y. W.; HUANG, S.M.; Growth of ZnO:Al films by RF sputtering at room temperature for solar cell applications, Solid-State Electronics, vol. 53, no. 11, pp. 1149-1153, 2009.

7. ABDALLAH, B.; JAZMATI,A.K.; REFAAI, R.; Oxygen Effect on Structural and Optical Properties of ZnO Thin Films Deposited by RF Magnetron Sputtering, Materials Research, vol. 20, no. 3, pp. 607-612, 2017 